\title{
Signal subspace based Enhancement and MAP Parameter Estimation of fMRI Signals
}

\author{
R.Srikanth, A.G.Ramakrishnan \\ Department of Electrical Engineering \\ Indian Institute of Science \\ Bangalore,'560012 INDIA \\ srikanth,ramkiag@ee.iisc.ernet.in
}

\author{
P.N.Jayakumar \\ Dept. Of Neuroimaging and Interventional Radiology \\ National Institute of mental Health and Neurosciences \\ Bangalore-560 029, India \\ jayakumarpn@nimhans.kar.nic.in
}

Abstract-- We propose a signal subspace based Functional Magnetic Resonance Image (fMRI) signal enhancement fol-: lowed by Maximum A Posteriori (MAP) estimation of parameters of Hemodynamic Response Function (HRF). The fMRI time-series, which is corrupted by physiological and scanner noise, is a low SNR signal. This signal is projected onto signal-plus-noise space and then enhanced in this space. The enhanced signal is then used to estimate the parameters of the HRF using MAP estimation. Preliminary results indicate that signal enhancement greatly improves the estimation performance.

\section{INTRODUCTION}

Magnetic Resonance Imaging (MRI) gives structural details of the organ imaged whereas functional MRI (fMRI) gives dynamics of the brain. fMRI is a noninvasive technique allowing for the evolution of brain processes to be dynamically followed in various cognitive or behavioral tasks. fMRI attempts to detect brain activity by localized, noninvasive measurements of the change in blood oxygenation called as BOLD (blood oxygenation level dependent) contrast. Measurements are collected under controlled conditions when subjects perform specific tasks prompted by some stimulus (e.g., motor task or visual task). There are two dominating design paradigms for $\mathrm{FMRI}$ experiments. In block design, subjects repeat the task for extended periods of time interleaved with similarly long periods of inactivity. In singletrial design (also called as event-related design), the task is performed only once, followed by inactivity (rest). This allows the full response evoked by the task to be analyzed. In applications like behavioral studies, where the task cannot be repeated, this design is very useful. We are interested in the analysis of single-trial fMRI data.

In the block design, the focus was on detecting the regions of activation [1], [4]. In single-trial design, we are interested in characterizing the BOLD signal [5], [6], [7]. A convenient way to analyze BOLD fMRI consists of considering the brain as a linear system characterized by its transfer function called as Hemodynamic Response Function (HRF). The HRF is the signal that BOLD fMRI would measure in response to a single; very short stimulus of unit intensity. Although several departures have been reported from linearity [6], it is be-

0-7803-7651-X/03/\$17.00 @20031EEs lieved that the linearity assumption holds in a wide range of experimental conditions. Estimation of the HRF is of great interest in analyzing fMRI data. It can give a deep insight into the underlying dynamics of brain activation and relationships between activated areas. For these studies, HRF can be modeled by parametric functions like Gaussian, Poisson, Gamma functions etc. The parameters such as mean (time to peak) signifies the delay in the response to activation; dispersion gives rise and fall rate of HR and the amplitude gives the strength of the response. In the next section, we give the details of the model. In this work, by assuming that the location of the activated regions is known, we estimate the parameters of the HRF using the maximum a posteriori (MAP) technique. We decide the prior required for the parameter, considering the experiment at hand.

Generally, the signal recorded during an fMRI experiment consists of colored noise with the required BOLD response. The noise is due to physiological sources such as pulse, breathing etc., and system sources like scanner and noise drift. The noise also depends upon the acquisition time or repetition time (TR) which decides the aliasing of the above noises and signal power. Generally signal to noise ratio (SNR) is very low. This poses difficulty in estimating the parameters of HRF. Hence a robust denoising technique is required to enhance the signal of interest which in turn would improve the parameter estimation. Generally band pass fil. tering [5] is used in reducing the noise component. In [4], a subspace based approach is proposed for a block design paradigm. The signal space is assumed to be spanned by fourier bases and noise subspace is estimated using the maximum likelihood estimation. Here, the motivation is to improve the detection of activation. In this work, we propose a subspace approach based on [8] and [9] for enhancing the fMRI signal. We then estimate the parameters of the HRF from the enhanced fMRI signal. This greatly improves the estimation performance. The performance of enhancement and estimation depends upon the sampling rate (TR).

\section{Model For HEMOdYNAMIC RESPONSE}

Hemodynamic response refers to the local change in blood oxygenation as an effect of increased neuronal activity. This change is not immediate but is delayed by $2-6$ seconds from the stimulus onset. It is observed that this response increases slowly and attains maximum and returns to the base line. Several models, both parametric and non parametric, have 


\section{Medical Image Processing / 521}

been proposed in the literature to characterize this response. Poisson function [1], Gaussian function [2], Gamma function [3] are some of the parametric models which parsimoniously model the HRF. In this work, we model HRF by Gaussian function. The proposed methods for signal enhancement and estimation can be applied for any HR model but the advantage of this model is that the parameters give a physiological interpretation [5]. The hemodynamic response as a function of time can be represented as:

$$
h(t)=\eta \exp \left(-(t . T R-\mu)^{2} / \sigma^{2}\right)
$$

where, $\mu$ represents the time lag from the onset of the stimuli to the peak of HR; $\sigma$ reflects the rise and decay time and $\eta$ denotes the amplitude of the response as shown in Figure 1 and $T R$ is the sampling period. Let $\theta=[\mu ; \sigma, \eta]$ denote the unknown parameters of the HR function. The response of the brain for a given task can be modeled as convolution of the HR function and the input task [6]. The input task can be considered as a binary function of time which has a value 1 during the period of task and a value of 0 during the rest period. Figure 2 shows the above convolution of $H R$ and the task. The observed fMRI time-series is corrupted by physiological noises like breath, heart beat, pulse and motion artifacts. This noise is in general colored and poses problem in enhancing and estimating the required response signal. The observed fMR signal can be modeled as:

$y_{n}(m)=\sum_{k} x(k) h_{n}(m-k)+w(m) \quad m=1 . . . T, n=1 \ldots N$

where, $y_{n}, x$ and $w$ are the observed fMR time-series of $n t h$ voxel, input task and noise component, respectively. $T$ and $N$ are respectively the total number of fMR scans and voxels.

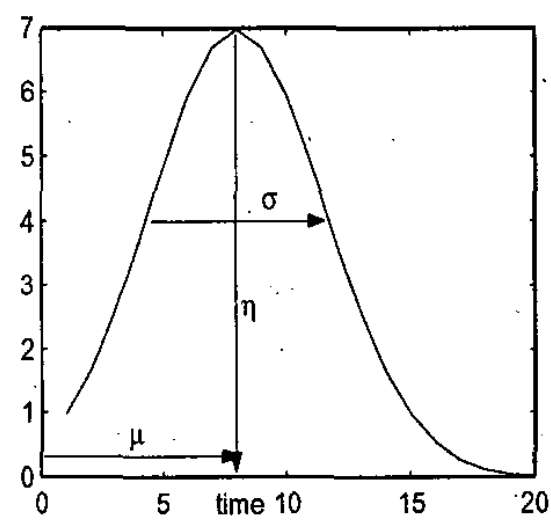

Figure 1. A sample HR function with parameters $\mu, \sigma, \eta$

\section{SigNAL ENHANCEMENT}

In this method, we assume that the original signal is confined to a subspace of the Euclidean space and the noise fills the
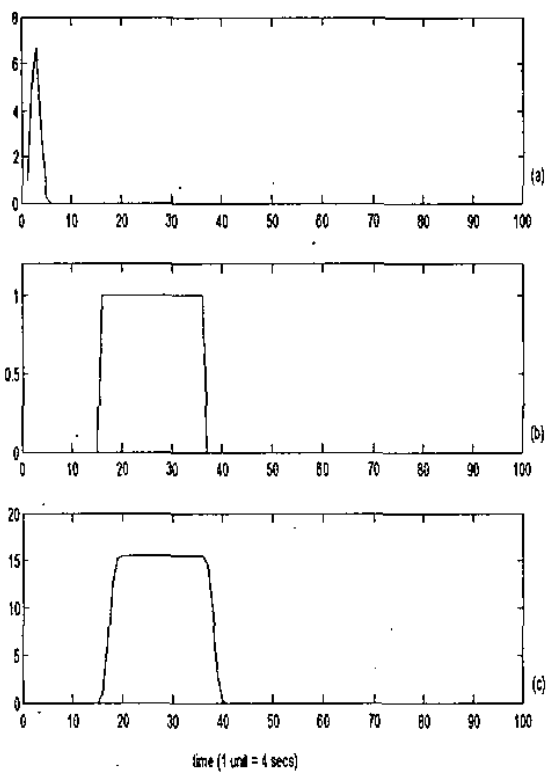

Figure 2. Signal model (a)HR function (b)Input activation profile (c) convolution of $\mathrm{HR}$ and input activation.

vector space of the noisy signal [8]. Hence, the vector space of the noisy signal is composed of a signal-plus-noise subspace and a complementary noise subspace. The signal-plusnoise subspace or simply the signal subspace, comprises vectors of actual signal and noise processes and noise subspace contains only the noise process. For enhancing the required signal buried in noise, the signal is projected onto the signal subspace and then the it is enhanced from this subspace. The model used in the subspace approach assumes that the noise signal is additive and uncorrelated with the signal of interest. The observed time series can be written as

$$
\mathbf{y}=\mathbf{z}+\mathbf{w}
$$

where $\mathbf{y}, \mathbf{z}$ and $\mathbf{w}$ are K-dimensional noisy fMR observation, convolution of HRF and input task and noise vectors, respectively. Let $\hat{\mathbf{z}}=H . \mathbf{y}$ be a linear estimator of the signal $\mathbf{z}$, where $H$ is a $K \times K$ matrix. The linear filter $H$ is designed in such a way that the energy in signal distortion is minimized subject to a constraint on the residual noise ie.,

$$
\epsilon=\hat{\mathbf{z}}-\mathbf{z}=(H-I) \cdot \mathbf{z}+H \cdot \mathbf{w}=\epsilon_{z}+\epsilon_{w}
$$

where $\epsilon, \epsilon_{z}$ and $\epsilon_{w}$ are observed signal residue, signal distortion and residual noise, respectively. Defining the energies of the signal distortion $E_{z}$ and the residual noise $E_{w}$ as

$$
\begin{gathered}
E_{z}=\operatorname{tr}\left(E\left[\epsilon_{z} \epsilon_{z}^{t}\right]\right) \\
E_{w}=\operatorname{tr}\left(E\left[\epsilon_{w} \epsilon_{w}^{t}\right]\right)
\end{gathered}
$$

where, ' $t$ ' is the transpose operator. We solve the following constrained optimization problem for the desired filter $H$ us- 


\section{TENCON $2003 / 522$}

ing Lagrange optimization [8],[9].

$$
\begin{gathered}
\min _{H} E_{z} \\
\text { subject to }: E_{w} / K \leq \sigma^{2}
\end{gathered}
$$

where $\sigma^{2}$ is a positive constant. The solution to the above equation is given by [9]

$$
H_{o p t}=R_{z}\left(R_{z}+\mu R_{w}\right)^{-1}
$$

where $R_{z}$ and $R_{w}$ are covariance matrices for the HR signal and noise, respectively and $\mu$ is the Lagrangian multiplier. We give the implementation details of this method in the following sections.

\section{Parameter Estimation}

After signal enhancement, we estimate the parameters $\theta$ of the HR function using maximum a posteriori estimation. Consider the signal model given by (2).

$$
\mathbf{y}_{\mathbf{e}}=\mathbf{z}_{e}+\mathbf{w}_{\mathbf{e}}
$$

where $\mathbf{y}_{\mathbf{e}}, \dot{z}_{\mathbf{e}}$ and $\mathbf{w}_{\mathbf{e}}$ are the T-dimensional vectors of enhanced signal, convolution of $\mathrm{HR}$ and input stimulation and residual noise, respectively and $\mathrm{T}$ is total number of scans. Let $\mathbf{w}_{\mathbf{e}}$ be a zero mean Gaussian random variable with covariance matrix $C$. The conditional density function of $\mathbf{y}_{\mathbf{e}}$ given $\theta$ is given by

$$
\begin{aligned}
p\left(\mathbf{y}_{\mathbf{e}} \mid \theta\right)=(2 \pi)^{-T / 2} \operatorname{det}(C)^{-1 / 2} & \\
& \quad \exp \left(-1 / 2\left(\mathbf{y}_{\mathbf{e}}-\mathbf{z}_{\mathbf{e}}\right)^{\mathbf{t}} \mathbf{C}^{-\mathbf{1}}\left(\mathbf{y}_{\mathbf{e}}-\mathbf{z}_{\mathbf{e}}\right)\right.
\end{aligned}
$$

We model the unknown parameters $\mu, \sigma$ and $\eta$ as independent Gaussian random variables.

$$
\begin{aligned}
p(\theta) & =p(\mu, \sigma, \eta)=p(\mu) p(\sigma) p(\eta) \\
\theta & \sim N\left(m_{\theta}, V_{\theta}\right)
\end{aligned}
$$

where $N()$ stands for gaussian probability density function, $m_{\theta}$ is the vector of means of gaussian random variables $\mu, \sigma$ and $\eta$ and $V_{\theta}$ is a diagonal matrix whose entries are variances of the above gaussian random variables. We find $\theta$ by maximizing the loglikelihood of posterior probability of $\theta$ given the observation $y_{e}$.

$$
\begin{aligned}
p\left(\theta \mid \mathbf{y}_{\mathbf{e}}\right) & =p\left(\mathbf{y}_{\mathbf{e}} \mid \theta\right) p(\theta) / p\left(\mathbf{y}_{\mathbf{e}}\right) \\
\log \left(p\left(\theta \mid \mathbf{y}_{\mathbf{e}}\right)\right) & =\log \left(p\left(\mathbf{y}_{\mathbf{e}} \mid \theta\right)\right)+\log (p(\theta))-\log \left(p\left(\mathbf{y}_{\mathbf{e}}\right)\right.
\end{aligned}
$$

The prior probability parameters $m_{\theta}$ and $V_{\theta}$ are chosen using the prior knowledge of the HRF. It is observed that when a stimulus of small duration (unit impulse) is applied, the HRF follows after some delay and attains peak after about $6 \mathrm{sec}-$ onds of application of the stimulus [5]. Also HRF lasts for a duration between 7 to 12 seconds for an unit impulse input. It is also observed that the amplitude of the response $\eta$ is around 4 units. This knowledge can be incorporated by choosing the means and standard deviations of $\mu, \sigma$ and $\eta$ as follows [5]:

$$
\begin{aligned}
\mu \sim N\left(m_{\mu}, \sigma_{\mu}\right) & =N(6,1.732) \\
\sigma & \sim N\left(m_{\sigma}, \sigma_{\sigma}\right)=N(2,1) \\
\eta \sim N\left(m_{\eta}, \sigma_{\eta}\right) & =N(4,1)
\end{aligned}
$$

where, $m_{\mu}, m_{\sigma}$ and $m_{\eta}$ are means of $\mu, \sigma$ and $\eta$ respectively; $\sigma_{\mu}, \sigma_{\sigma}$ and $\sigma_{\eta}$ are standard deviations of $\mu, \sigma$ and $\eta$ respectively. Therefore, the prior probability $\theta$ can be specified as:

$$
\begin{aligned}
\theta & \sim N\left(m_{\theta}, V_{\theta}\right), \text { where } \\
m_{\theta} & =\left[m_{\mu}, m_{\sigma}, m_{\eta}\right]^{t}=[6,4,2]^{t} \\
V_{\theta} & =\operatorname{diag}\left(\sigma_{\mu}^{2}, \sigma_{\sigma}^{2}, \sigma_{\eta}^{2}\right)=\operatorname{diag}(3,1,1)
\end{aligned}
$$

\section{SIMULATION RESULTS}

We use the fMRI data generated in National Institute of Mental Health and Neuro Sciences (NIMHANS), Bangalore. The time-series of fMRI volume is acquired at every 3 seconds $(T R=3)$. To verify the performance of the above methods, we choose a patch of $10 \times 10$ from a slice where there is no activation. We add the convolution of Gaussian signal of known parameters with input stimulation signal (boxcar function) to the time-series of voxels at known locations. We add two synthetic HRFs with parameters $\theta_{1}=[5.5,3.2,3.2]$ and $\theta_{2}=[7,2.5,4.7]$ at locations indicated in figure 3 . Now the task is to enhance these signals and estimate the parameters of the HR functions. We do not consider the first four samples in every voxel time-series to account for the scanner saturation effects. We assume that the rest condition is from frame $t=1$ to $t=60 \mathrm{secs}$. and activation condition from $t=60$ to $t=120 \mathrm{secs}$ followed by again rest condition from $t=120$ to $t=300 \operatorname{secs}(\mathrm{N}=100)$ ie.,

$$
\begin{aligned}
x(t) & =1, \quad t \in(60,120) \\
& =0, \text { otherwise }
\end{aligned}
$$

Before applying the signal enhancement filter, mean of each pixel time-series is removed. This amounts to removing the structural details of the fMR image. We estimate the noise covariance matrix $R_{w}$ from the fmri time-series where there is no HRF. Since the activation is between the scans 20 $(t=60)$ and $40(t=120 \mathrm{secs})$, the samples from $y(1: 20)$ and $y(46: 100)$ at each voxel can be used for estimating $R_{w}$. Similarly, signal-plus-noise covariance matrix $R_{y}$ is estimated from the samples $y(21: 45)$ at each voxel. Since the noise and signal are uncorrelated, the covariance matrix of signal $R_{z}$ is $R_{y}-R_{w}$. We choose $\mu=3$ based on the experimental results. We divide the voxel time-series into frames of 16 points with a 50 percent overlap. Hence $R_{z}$ and $R_{w}$ would be $16 \times 16$ matrices. We apply the filter $H_{\text {opt }}$ to the frames and the enhanced signal vectors are hamming windowed and combined using overlap and add method. Figures 4 and 5 respectively show the original and enhanced signals at two voxels with parameters $\theta_{1}$ and $\theta_{2}$. We then apply the MAP estimator to estimate the parameter $\theta$. Figure 6 shows the original and the estimated signals using MAP and maximum likelihood (ML) estimators. Clearly, MAP gives a good estimation provided prior is chosen appropriately.

\section{CONCLUSIONS}

We presented a new signal subspace based approach for fMRI signal enhancement. This involves projecting a fMRI signal 


\section{Medical Image Processing / $\mathbf{5 2 3}$}

onto the signal-plus-noise subspace and then enhancing the signal in this space by a statistical linear filter. The enhanced signal is then used for parameter estimation of hemodynamic response function. We use MAP estimator for parameter estimation choosing a proper prior for the parameter. We compare estimation using MAP with Maximum Likelihood Estimation (MLE). MAP gives superior performance over MLE. Preliminary results suggest that signal enhancement greatly improves the estimation performance.

\section{ACKNOWLEDGEMENTS}

We thank Prof.P.N.Jayakumar of NIMHANS for providing the required data. We also thank Dr.Bapiraju, Pammi Chandrasekhar and Krishna Prasad of University of Hyderabad for their help and discussion.

\section{REFERENCES}

[1] K.J.Friston, P.Jezzard and R.Turner, "The analysis of functional MRI time-series", Human Brain Mapping, vol.1, pp.153-174, 1994.

[2] J.C.Rajapakse, F.Kruggel, J.M.Maisog and D.Y.Voncramman, "Modeling hemodynamic response for analysis of functional MRI time-series", Human Brain Mapping, vol.6, pp.283-300, 1998.

[3] G.M.Boynton, S.A.Engel, G.HGlover and D.J.Hegger, "Linear system analysis of functional magnetic resonance imaging in human. ", J.Neurosci., vol.16,no.13, pp.4207-4221, 1996.

[4] Babak A.Ardekani, Jeff Kershaw, Kenichi Kashikura and Iwao Kanno, "Activation Detection in Functional MRI Using Subspace Modeling and Maximum Likelihood Estimation", IEEE trans. Medical Imaging, vol.18,no.2, pp.101-114, 1999.

[5] Markus Svensen,Frithjof Kruggel and D.Yves von Cramon, "Probabilistic Modeling of Single-trial fMRI Data", IEEE trans. Medical Imaging, vol.19,no.1, pp.25-35, 2000 .

[6] Cyril Goutte, Finn Arup Nielsen and Lars Kai Hansen, "Modeling the Haemodynamic Response in fMRI Using Smooth FIR filters", IEEE trans. Medical Imaging, vol.19,no.12,pp.1188-1201, 2000.

[7] G.Marrelec, H.Benali, P.Ciuliu and J.B.Pioline, "Bayesian Estimation of the Hemodynamic Response Function in Functional MRI", 21st International Workshop, American Institute of Physics, pp. 229-247, 2002.

[8] Yariv Ephraim, Harry L.Van Trees, "A Signal Subspace Approach for Speech Enhancement", IEEE trans. Speech and Audio Process., vol.3,no.4, pp.251-266,
1995.

[9] Yi Hu and Philipos C.Loizou "A Subspace Approach for Enhancing Speech Corrupted by Colored Noise", Proc. ICAASP, vol.1, pp.573-576, 2002.
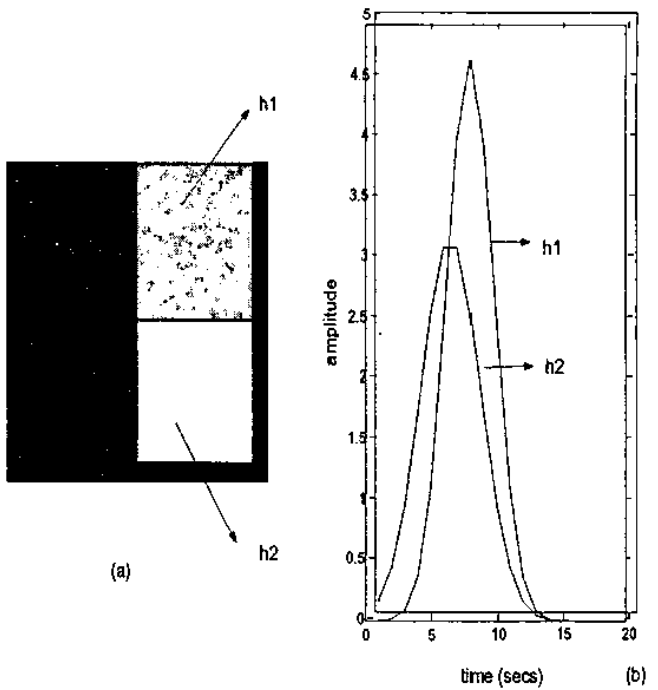

Figure 3. (a) Activation Profile (b) Original HRFs at indicated locations
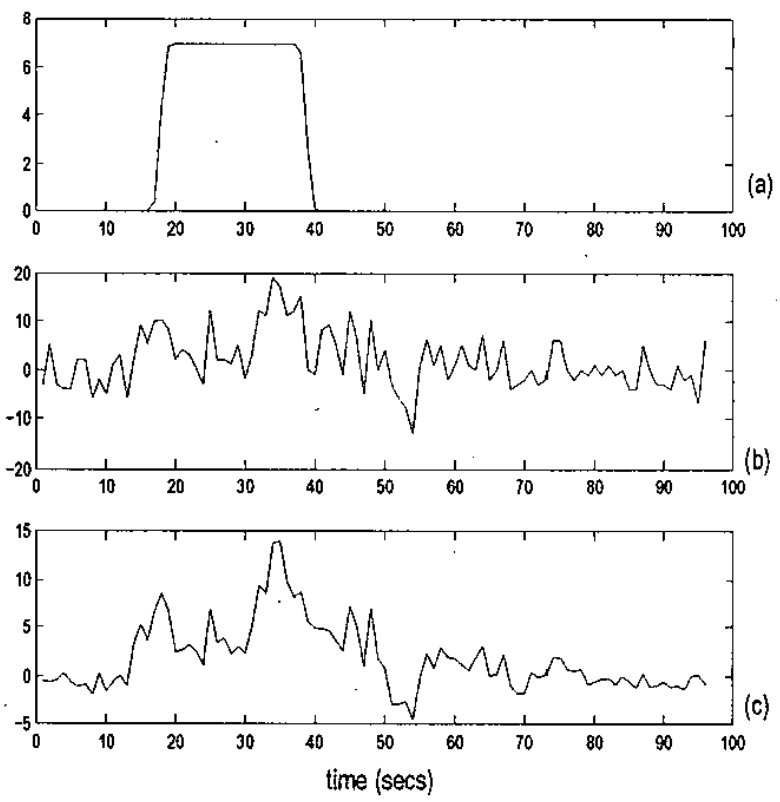

Figure 4. Signal enhancement with parameter $\theta_{1}$ using subspace method. (a) Original fMRI Signal (simulated), (b)fMRI signal in real noise (c)Signal enhanced by subspace method 

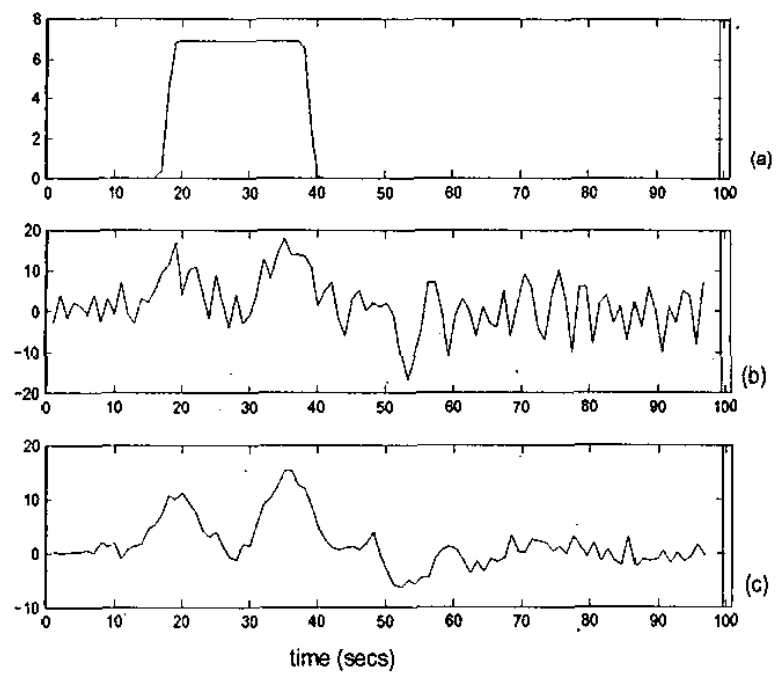

Figure 5. Signal enhancement with parameter $\theta_{2}$ using subspace method. (a) Original fMRI Signal (simulated), (b) fMRI signal in real noise (c) Signal enhanced by Subspace method
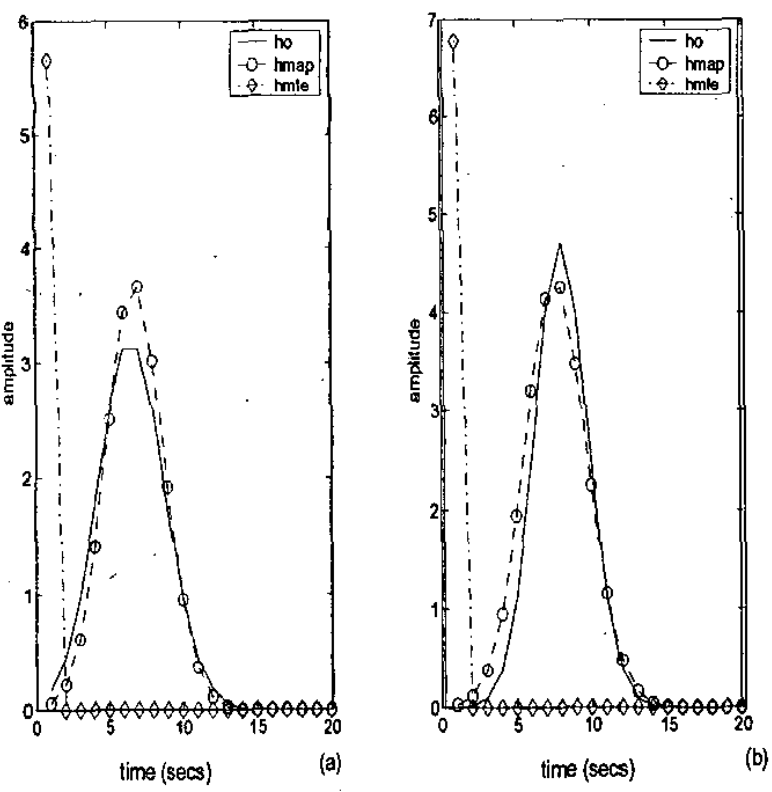

Figure 6. HRF estimated by MAP and MLE (a) HRF with parameter $\theta 1$ (b) HRF with parameter $\theta 2$ 